\title{
National cost savings from an ambulatory program for low-risk febrile neutropenia patients in Australia
}

\author{
Michelle Tew ${ }^{1,2,5} \mathrm{MPH}$, MPharm, PhD Candidate \\ Daniel Forster ${ }^{1}$ BPhysio, MBBS, MPH, Infectious Diseases Advanced Trainee \\ Benjamin W. Teh ${ }^{2,3,4}$ MBBS, PhD, FRACP, Infectious Diseases Physician \\ Kim Dalziel ${ }^{1} \mathrm{PhD}$, Associate Professor of Health Economics \\ ${ }^{1}$ Centre for Health Policy, Melbourne School of Population and Global Health, 207 Bouverie Street, Carlton, \\ Vic. 3053, Australia. Email: dpforster@gmail.com; kim.dalziel@unimelb.edu.au \\ ${ }^{2}$ National Centre for Infections in Cancer, Peter MacCallum Cancer Institute, 305 Grattan Street, Melbourne, \\ Vic. 3000, Australia. Email: Ben.Teh@petermac.org \\ ${ }^{3}$ Department of Infectious Diseases, Peter MacCallum Cancer Institute, 305 Grattan Street, Melbourne, \\ Vic. 3000, Australia. \\ ${ }^{4}$ Sir Peter MacCallum Department of Oncology, Peter MacCallum Cancer Institute, 305 Grattan Street, Melbourne, \\ Vic. 3000, Australia. \\ ${ }^{5}$ Corresponding author. Email: Michelle.Tew@unimelb.edu.au
}

\begin{abstract}
Objective. The management of low-risk febrile neutropenia (FN) patients through ambulatory programs has demonstrated comparative safety and effectiveness to in-patient strategies. However, there is limited evidence of benefits of changing practice, particularly on a national scale. The aim of this study was to estimate costs and benefits of the program over a 10 -year time horizon.

Methods. A comparative cost analysis from a health system perspective was performed, comparing costs and length of stay (LOS) of patients enrolled in an ambulatory program to a historical cohort who did not receive the program. Generalised linear models were used for analysis and bootstrapped to account for uncertainty. National data of identified FN admissions were used to inform future projections, with varying proportions of low-risk patients and eligibility for the ambulatory program.

Results. The overall LOS for patients in ambulatory cohort was 1.9 days shorter (95\% confidence interval (CI) 1.0-2.8 days), a 50\% reduction in in-patient bed-days. Although patients in the ambulatory cohort incurred additional costs due to care received outside hospital (mean $( \pm$ s.d.) A $\$ 828.03 \pm 124.30)$, the mean total cost incurred remained substantially lower than that of the historical cohort (A \$2979 lower; 95\% CI A \$772-5391). On a national scale, this could translate into A \$62.7 million in costs averted and 41347 bed-days saved over 10 years if the low-risk prediction rate and eligibility for ambulatory programs remained at currently observed rates.

Conclusions. The wider implementation of a safe and effective ambulatory program to manage low-risk FN patients can result in significant return-on-investment for the healthcare system by eliminating avoidable costs due to unnecessary lengthy hospital admissions.

What is known about the topic? There is strong evidence demonstrating out-patient treatment of low-risk FN patients to be an effective and cost-effective strategy compared with continued in-patient hospitalisation.

What does this paper add? This study demonstrates the sustainability of the ambulatory program in ensuring cost benefits and in-patient beds through real-life implementation data. It also provides evidence of the substantial cost and bed-days potentially averted when the cost savings and difference in LOS are estimated on a national scale over a 10-year time horizon. What are the implications for practitioners? The management of low-risk FN patients through ambulatory or outpatient programs is a safe and effective approach. There is strong evidence demonstrating the likely cost savings and considerable bed-days saved, which can be reallocated to meet other medical demands.
\end{abstract}

Additional keywords: national projections.

Received 13 March 2019, accepted 28 May 2019, published online 17 September 2019 


\section{Introduction}

Febrile neutropenia (FN) is a common and potentially lifethreatening complication for cancer patients undergoing chemotherapy that necessitates prompt treatment. Historically, this involved in-hospital management with broad spectrum intravenous (i.v.) antibiotics. ${ }^{1}$ However, it is recognised that FN patients are a heterogeneous population, whereby only a small proportion of FN patients are at high risk of complications or death. ${ }^{2}$ Therefore, not all $\mathrm{FN}$ patients necessarily require in-patient care. The Multinational Association of Supportive Care in Cancer (MASCC) risk index ${ }^{2}$ is a validated clinical tool that has been successfully used to identify FN patients with a low risk of complications. Clinical studies have shown that low-risk FN patients can be successfully treated with oral instead of i.v. antibiotics without compromising patient safety. ${ }^{3,4}$ The management of these patients through ambulatory or out-patient programs has also demonstrated comparative safety and effectiveness compared with in-patient management strategies. ${ }^{5-7}$ In addition to having a shorter length of hospital stay (LOS), the benefits of out-patient care include improved health-related quality of life, ${ }^{8,9}$ reduced risk of hospital-acquired infections and lower costs. ${ }^{10-13}$

There is consistent evidence demonstrating out-patient treatment of low-risk FN patients to be a cost-effective strategy compared with continued in-patient hospitalisation. ${ }^{12,14}$ Although the advantages of ambulatory programs are evident, there remain inconsistencies in managing these patients. ${ }^{15-17}$ Patient willingness, suitability of home environment and/or prevailing medical condition have been identified as possible reasons for the low uptake. ${ }^{16,18}$ Clinician acceptance has also been recognised as a potential barrier. ${ }^{19}$ Although much is known about the potential barriers that could explain the slow uptake, there is limited evidence of the potential benefits of changing practice, particularly on a national scale. A better understanding of the implications of different management strategies is increasingly important to inform healthcare resource allocation decisions in a budget-constrained environment.

This study builds upon an existing evaluation of an ambulatory program implemented at the Peter MacCallum Cancer Centre (PMCC), a tertiary cancer centre in Australia. The PMCC ambulatory program for $\mathrm{FN}$ patients is a nurse-led model of care with MASCC risk assessment performed for patients presenting with FN. Patients were recruited into the program following three stages of evaluation: (1) risk stratification using the MASCC risk index (low risk defined as scores $\geq 21$ ); (2) suitability for switching from i.v. to oral antibiotics; and (3) suitability for early discharge, after at least one dose of i.v. antibiotics and 24-hour observation. Patients in the ambulatory program are discharged with a course of oral antibiotics, followed-up by ambulatory care nurses and reviewed by an infectious disease physician within 1 week after discharge. Fig. 1 shows a representation of the evaluation process. In its first year of implementation, the early discharge of eligible low-risk FN patients reduced in-patient LOS from 4.0 to 1.1 days, resulting in 72.5 in-patient bed-days saved across 25 patients; this translated into a net cost reduction of A $\$ 71895$ after accounting for implementation and operational costs. Four of 25 patients (16\%) required readmission, and no deaths were reported. A detailed description of the program,

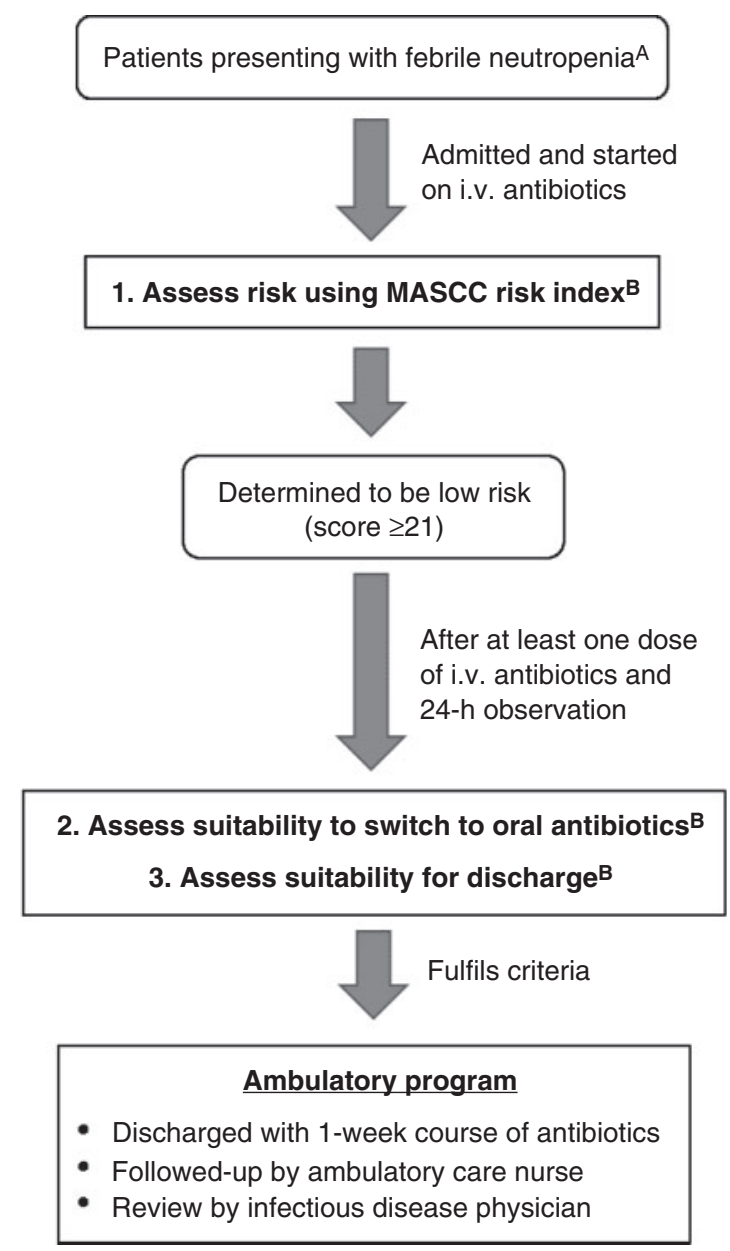

Fig. 1. Diagrammatic representation of the evaluation process for ambulatory program eligibility. ${ }^{\mathrm{A}} \mathrm{Febrile}$ neutropenia is defined as fever $\geq 38.3^{\circ} \mathrm{C}$ or $\geq 38.0^{\circ} \mathrm{C}$ on two occasions and an absolute neutrophil count of $<1.0 \times 109$ cells L ${ }^{-1}$. ${ }^{\mathrm{B}}$ Assessment using the Multinational Association of Supportive Care in Cancer (MASCC) risk assessment and eligibility screening tool published in Teh et al. ${ }^{11}$

including its implementation, safety and cost in the first year has been reported by Teh et al. ${ }^{11}$

The aims of the present study were to demonstrate the sustainability of the program in ensuring cost benefits and to model the potential cost averted and in-patient bed-days saved over 10 years if the program is rolled out nationwide. Information from this study will assist decision making by clinicians and policy makers by providing estimates of the economic impact of introducing ambulatory care programs as standard of care across Australia to manage low-risk FN patients.

\section{Methods}

\section{Cost analysis}

A cost analysis from the healthcare perspective was performed comparing patients' costs and LOS. Patients were prospectively enrolled in the ambulatory program between March 2014 and February 2017. These patients were compared to a historical cohort of retrospectively identified consecutive FN patients from 
February to July 2011 who were assessed to be low-risk using the MASCC risk index and fulfilled eligibility criteria to switch to oral antibiotics and be discharged into a theoretical ambulatory program. ${ }^{11}$ These patients were identified from medical records. Patients in the historical cohort were subjected to the standard of care before the implementation of the ambulatory program, which consisted of hospital admission of all FN patients with a course of i.v. antibiotics until resolution of fever and neutropenia. In-patient admission costs were calculated based on each patient's Australian refined diagnosis-related groups (AR-DRGs) ${ }^{20}$ and LOS.

Ambulatory care costs were estimated based on the components of resource used. This included staff time spent on home nursing visits, follow-up telephone call by the nurse coordinator, consultation to develop an appropriate follow-up protocol by an infectious disease physician, physician and nurse time required to review the patient in a specialist clinic, two sets of blood tests and a patient discharge information pack. Patients in the ambulatory cohort were also discharged with a prescription for a 1 -week standard course of antibiotics. These patients were followed-up for the duration of the ambulatory program (7 days) and readmitted patients were treated with i.v. antibiotics and their LOS recorded.

Generalised linear models (GLM) were used to analyse cost and LOS. For costs, the gamma family of distributions was determined using the modified Park test and its log link determined using Pearson correlation, Pregibon and modified Homer and Lemeshow tests, whereas for LOS, Poisson distribution with $\log$ link was used. ${ }^{21,22}$ Age, sex and cancer types were included in the models to control for possible baseline imbalances. The choice of models was also based on the Akaike information criterion (AIC). To account for sampling uncertainty, sensitivity analysis was undertaken using bootstrapping with 1000 replications using the recycled predictions method. ${ }^{22}$

\section{Projected cost and bed-days averted}

National data of all FN hospital admissions among cancer patients aged $\geq 15$ years between 2009 and 2014 were obtained from the Independent Hospital Pricing Authority (IHPA), a national government agency responsible for collecting and reporting hospital use and expenditure data. The selection criteria for FN patients as described by Lingaratnam et al. ${ }^{23}$ were used. The data captured all FN in-patient episodes, regardless of risk types, and were used to inform on future trends for hospital presentation with FN.

The proportion of low-risk FN patients varies internationally. A review of the 10-year use of MASCC index reported low-risk prediction rates in the range of $70-75 \%$ across several international studies, ${ }^{13}$ whereas Australian studies evaluating early discharge strategies indicated a $56-65 \%$ low-risk prediction, with up to $41 \%$ of these episodes subsequently converted to ambulatory care. ${ }^{15,24}$ Therefore, in-patient bed-days and cost averted over 10 years were calculated using bootstrapped results with proportions of low risk (LR) ranging from $50 \%$ to $80 \%$, and $30 \%$ to $60 \%$ eligibility for the ambulatory program (EA).

All costs are expressed in 2017 Australian dollars adjusted using the Consumer Price Index from the Australian Bureau of Statistics $^{25}$ and discounted at 5\% annually as per Australian recommendations. ${ }^{26}$ All data were analysed using STATA version 14.0 (StataCorp, College Station, TX, USA).

Unless indicated otherwise, data are presented as the mean \pm s.d.

\section{Ethics approval}

This study was approved by the Peter MacCallum Cancer Centre Ethics Committee.

\section{Results}

Between March 2014 and February 2017, 50 low-risk FN patients were enrolled into the ambulatory program ( 25 patients in first year, 25 patients in the second and third years). The baseline characteristics of patients in the ambulatory and historical cohorts are given in Table 1 . Both cohorts were well balanced, except for sex, where there was a lower proportion of males in the ambulatory program compared with the historical cohort $(P=0.016)$. Of the 50 patients in the ambulatory cohort, five hospital readmissions $(10 \%)$ were recorded and no deaths were reported. Time to readmission was approximately $1.4 \pm 0.7$ days.

\section{Cost analysis}

A breakdown of the components, program protocol, quantity or time required and costs of resource used for the ambulatory program is provided in Table 2 . The mean cost of providing ambulatory care outside of hospital over a 1-week period was A $\$ 828.03 \pm 124.30$ per patient.

A comparison of LOS and total cost between the two cohorts is presented in Table 3. Patients in the ambulatory cohort had a significantly shorter length of initial hospital admission of 2.1 days (95\% confidence interval (CI) $1.3-2.9 ; P<0.001)$. Four patients were readmitted to the same hospital and their average LOS ranged from 0.7 to 5.6 days. Overall, patients in the ambulatory cohort had a total LOS of $1.9 \pm 1.7$ days. This was 1.9 days shorter (95\% CI $1.0-2.8$ days shorter; $P<0.001)$ than patients in the historical cohort, indicating a $50 \%$ reduction in inpatient bed utilisation as a result of the early discharge protocol. Mean total cost incurred by the ambulatory cohort remained lower than the historical cohort. The cost difference between the two cohorts was A $\$ 2839$ (95\% CI \$949-4730; $P=0.004)$.

Bootstrapped results from both GLM regressions are presented in Table 3. These estimates closely reflect those from the direct comparison analysis. However, the bootstrapped results for total cost difference yielded a wider $95 \%$ CI.

Table 1. Characteristics of patients across the two cohorts Data are given as the mean \pm s.d. or as $n(\%)$

\begin{tabular}{lccc}
\hline Patient characteristics & $\begin{array}{c}\text { Ambulatory } \\
\text { cohort }(n=50)\end{array}$ & $\begin{array}{c}\text { Historical cohort } \\
(n=27)\end{array}$ & $P$-value \\
\hline Age (years) & $49.2 \pm 15.7$ & $50.1 \pm 18.2$ & 0.819 \\
Male sex & $19(38.0)$ & $18(66.7)$ & 0.016 \\
Malignancy type & & & 0.982 \\
$\quad$ Haematological & $11(22.0)$ & $6(22.2)$ & \\
$\quad$ Solid organ & $39(78.0)$ & $21(77.8)$ & \\
\hline
\end{tabular}


Table 2. Components and associated costs of the ambulatory program

AMA, Australian Medical Association; DoH, Australian Government Department of Health

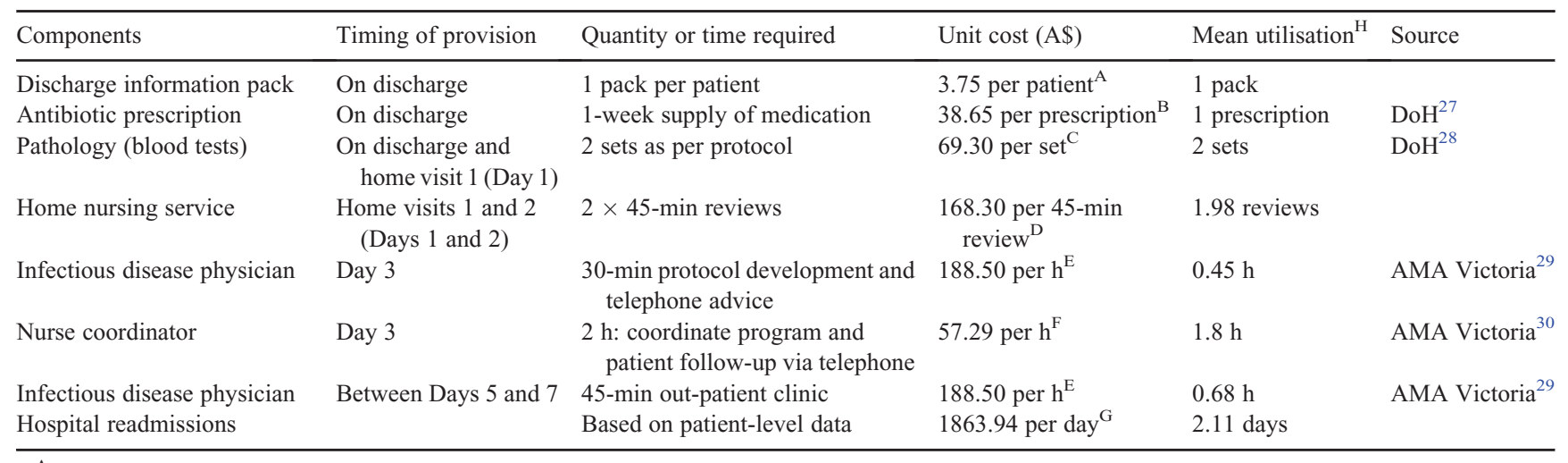

${ }^{\mathrm{A}}$ Calculated based on market price.

${ }^{B}$ Prescription for amoxicillin-clavulanic acid (875/125 mg, b.d.) and ciprofloxacin (750 mg, b.d.).

${ }^{\mathrm{C}}$ Medicare Benefit Scheme item numbers 65070 and 66512.

${ }^{\mathrm{D}}$ Based on hospital administrative records.

${ }^{\mathrm{E}}$ Based on hourly rates of a Year 2 specialist $+30 \%$ overhead cost.

${ }^{\mathrm{F}}$ Based on hourly rates of a Registered Nurse Grade $4 \mathrm{~A}$ Year $2+30 \%$ overhead cost.

${ }^{\mathrm{G}}$ Based on mean in-patient cost of ambulatory cohort.

${ }^{\mathrm{H}}$ Average resource used per patient.

Table 3. Comparison of length of stay and total cost between cohorts

Unless indicated otherwise, data are given as the mean \pm s.d. CI, confidence interval

\begin{tabular}{|c|c|c|c|c|}
\hline & Ambulatory cohort $(n=50)$ & Historical cohort $(n=27)$ & Difference $(95 \% \mathrm{CI})$ & $P$-value \\
\hline \multicolumn{5}{|l|}{ Length of stay (days) } \\
\hline Initial hospital admission & $1.7 \pm 1.5$ & $3.8 \pm 2.1$ & $2.1(1.3-2.9)$ & $<0.001$ \\
\hline Readmission $^{\mathrm{A}}$ & $2.1 \pm 2.3$ & - & & \\
\hline Total length of stay & $1.9 \pm 1.7$ & $3.8 \pm 2.1$ & $1.9(1.0-2.8)$ & $<0.001$ \\
\hline Bootstrapped $( \pm \text { s.e.m. })^{B}$ & $1.92 \pm 0.23$ & $3.89 \pm 0.43$ & $1.96(1.00-2.95)$ & \\
\hline \multicolumn{5}{|l|}{ Cost (A\$) } \\
\hline Initial hospital admission & $3293.3 \pm 3106.1$ & $7354.0 \pm 4907.9$ & 4060.7 (2239. 7-5881.7) & $<0.001$ \\
\hline Ambulatory care cost & $828.0 \pm 124.3$ & - & & \\
\hline Readmission & $3933.3 \pm 3782.3$ & - & & \\
\hline Total cost & $4514.7 \pm 3374.8$ & $7354.0 \pm 4906.9$ & $2839.3(948.9-4729.7)$ & 0.004 \\
\hline Bootstrapped $( \pm \text { s.e.m. })^{B}$ & $4493.65 \pm 463.57$ & $7472.58 \pm 1034.17$ & $2978.93(771.85-5390.96)$ & \\
\hline
\end{tabular}

${ }^{\mathrm{A}}$ Based on four patient readmissions. One patient was readmitted to a different hospital, therefore length of stay was undetermined. Missing data was imputed with mean length of readmission.

${ }^{\mathrm{B}}$ Results from general linear model regressions, bootstrapping with 1000 replications.

\section{National projections}

Data obtained from IHPA showed an average increase of FN hospitalisation episodes by approximately $4 \%$ annually, increasing from 7350 in 2010 to 8708 in 2015 . Assuming this increase remains constant over the next 10 years, it is estimated that by 2020 and 2025 the number of FN episodes would increase to 10318 and 12012, respectively. Fig. 2 shows the actual and projected number of hospitalisation episodes of FN in Australia.

The estimated cumulative bed-days and cost averted over 10 years would vary depending on the LR prediction rates and the proportion of low-risk EA patients. Fig. 3 shows the range of costs averted and bed-days saved over 10 years if the ambulatory program were to continue to produce a cost saving of A $\$ 2979$ per patient. Based on the best available Australian evidence, ${ }^{15,24}$ for a scenario reflecting an LR prediction rate of $60 \%$ and $40 \%$ EA, the estimated discounted total cost averted over 10 years would

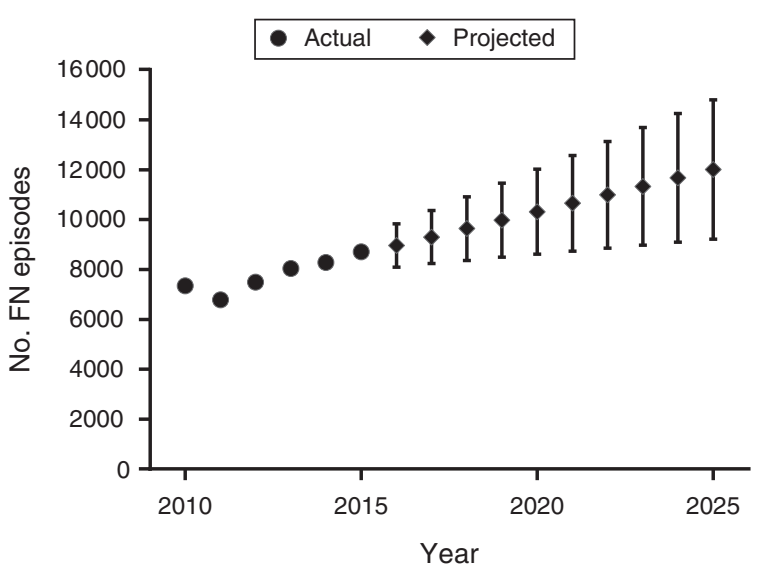

Fig. 2. Actual and projected number of hospitalisation episodes of febrile neutropenia (FN) in Australia. 
(a)

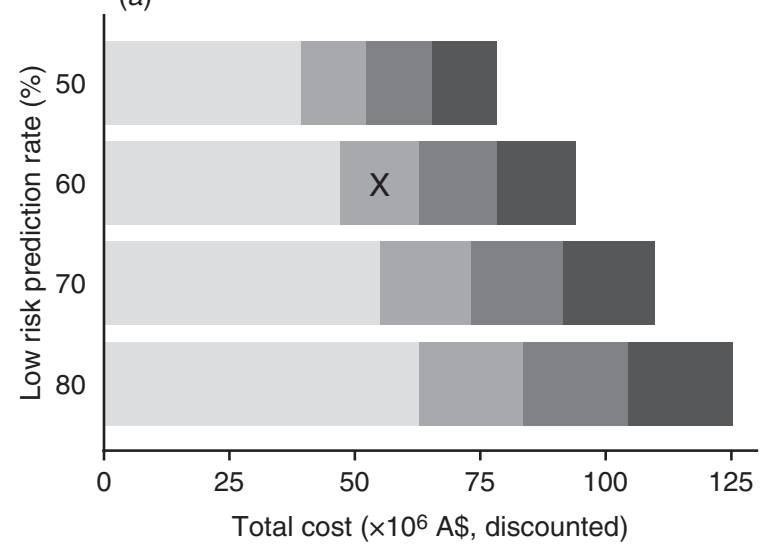

(b)

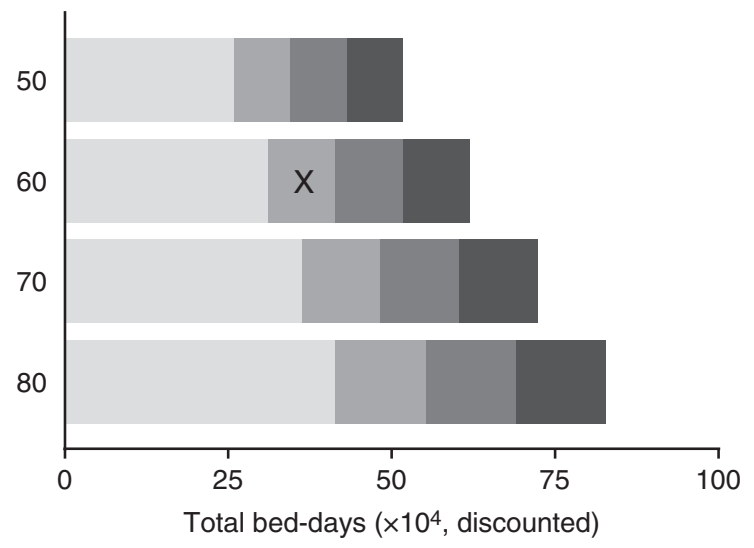

$\%$ Eligibility for ambulatory program

$30-40=50=60$
-50

Fig. 3. Estimated cumulative $(a)$ cost averted and $(b)$ bed-days saved over 10 years by the percentage identified as low risk (LR) febrile neutropenia and by the percentage eligible for the ambulatory program (EA). The ' $\mathrm{X}$ ' represents estimates based on best-available Australian evidence.

be A $\$ 62.7$ million (95\% CI A\$16.2-113.4 million). This cost is associated with a cumulative total of 41347 (95\% CI 21 137-62 130) bed-days saved.

\section{Discussion}

The ambulatory program to manage low-risk FN patients in an out-patient setting at PMCC represents a real-world implementation of the program with proven effectiveness and has demonstrated sustained cost benefits to the health system since it was implemented in 2014. The safety of patients was not compromised, with an overall readmission rate of $10 \%$ and no deaths reported. On average, the ambulatory program cost A $\$ 828$ per patient for care provided in their homes and follow-up consultations. Despite this, the mean total cost of the ambulatory cohort was A $\$ 2979$ (per patient) lower than care delivered to the historical cohort, even after taking into account readmission costs. This cost saving was driven primarily by the shorter LOS due to early discharge.

In Australia, the cost of managing FN is considerable. In 2015, it was estimated that there were 8708 in-patient episodes of FN among cancer patients, totalling an estimated A\$251 million (data provided by the IHPA). This estimate captures all FN episodes regardless of risk type and equated to A $\$ 28801$ per episode with an average LOS of 14.8 days. This is similarly observed in the US, where a large study conducted in 2010 assessing the economic burden of FN-related hospitalisation among cancer patients showed a mean hospitalisation cost of US\$18 880. ${ }^{31}$ The lower cost observed in the US study is likely due to the inclusion of less severe cancer groups (leukaemia and myeloma were excluded), hence the shorter LOS. Nonetheless, the mean costs per day of hospitalisations were comparable. It is evident that the economic burden of hospitalisations related to FN is significant and is not unique to Australia. It is also widely recognised that a large proportion of FN patients is of low risk; ${ }^{13-15}$ therefore, there is scope to reduce the national average
LOS of FN patients and alleviate the cost burden through the implementation of effective and safe strategies such as early discharge programs for low-risk FN patients.

The present study has demonstrated, for the first time, the substantial cost and bed-days potentially averted when the cost savings and difference in LOS are estimated at a national scale. Most cost studies have primarily focused on the delivery of ambulatory care and its comparison to an existing standard of care (in-patient strategy). The strengths of the present study include real-life implementation data and the use of national statistics to inform future projections. The estimated total cost and bed-days averted over 10 years is A $\$ 62.7$ million and 41347 respectively if the LR prediction rate and proportion converted to ambulatory programs remained at the current rate as indicated by local studies. Despite the wide $95 \%$ CIs, the ambulatory program remains a cheaper and more effective option than in-patient management of these low-risk patients, and is likely to translate well to other centres nationally.

The present cost analysis adds to the growing literature demonstrating out-patient treatment strategies to manage lowrisk FN patients to be cost-effective approaches compared with in-patient management. Although this study was conducted in the Australian setting, similar early discharge programs have been implemented elsewhere; therefore, these results could be applied internationally. In the present study, a mean reduction of 2 in-patient days and potential cost savings of A \$2979 per patient ( $40 \%$ reduction in cost) were observed. A randomised controlled single-centre study conducted in the UK also reported a reduction of 2 in-patient days and that out-patient treatment was $44 \%$ cheaper, ${ }^{14}$ whereas a US study reported a larger difference of 4.4 days and a $49 \%$ cost reduction. ${ }^{8}$ Potential cost-savings were estimated to be up to $55 \%$ in an economic modelling analysis in the Canadian setting. ${ }^{12}$ As such, the potential cumulative beddays and cost averted could possibly be larger in these countries.

It is acknowledged that such ambulatory programs would require significant initial investments to ensure successful 
implementation, including institutional support for the required infrastructure, a committed multidisciplinary team and welldefined protocols to manage patient monitoring and follow-up. ${ }^{32}$ Furthermore, funding to ensure sustainability of a program often also requires sound evidence and justification. The projected cost savings demonstrated at a national scale in the present study provide a strong case for institutions and healthcare policy makers in making resource allocation decisions for the ambulatory program. In addition, efficiencies in running the program could translate into higher adoption rates and thus greater cumulative cost and bed-days saved; for example, a 10\% increase in the proportion eligible for the ambulatory program above the current $40 \%$ would result in an additional A\$15.7 million and 10337 bed-days saved (Fig. 3).

It is recognised that this study has several limitations. The small sample size of this study is an important limitation subject to biases and inadequate statistical power. Although the estimated cost and bed-days averted are consistent with findings in the literature, $8,12,14,15$ it is acknowledged that there is a large amount of uncertainty around the extrapolated estimates based on small sample size. As such, the wide CIs should be taken into account when interpreting the results. It is also acknowledged that the use of a historical cohort can have an effect on the economic analysis because changes in practice can occur over time, affecting resource use. However, hospitalisation costs were calculated based on each patient's AR-DRG and LOS and thus likely overcome this issue. Although there was a disproportionate distribution by sex, results from the regression analysis (GLM) did not indicate any significant differences between males and females in the cost and LOS outcomes. There was a drop in the number of patients recruited for the ambulatory program in the second and third years $(n=25)$ compared with first year $(n=25)$. Although this can be largely ascribed to the discontinuity in funding a dedicated nurse to help with patient recruitment after the first year, other factors, such as patient and physician willingness, medical concerns and psychosocial factors, could potentially also have had an effect. ${ }^{15,16,24}$ In light of the substantial potential savings in terms of costs and bed-days demonstrated in this study, considerations to allocate resources to implement and support the continuity of an ambulatory program to manage low-risk FN patients is warranted.

\section{Conclusions}

The economic burden of hospitalisations related to $\mathrm{FN}$ is significant. The management of low-risk patients through ambulatory or out-patient programs is a safe and effective approach. Further, there has been consistent evidence to demonstrate the likely cost savings. A national roll-out of an ambulatory program across Australia could result in up to A $\$ 62.7$ million cost averted and 41347 bed-days saved over 10 years if the LR prediction rate and EA remained at the current rate.

\section{Competing interests}

Benjamin Teh has received speaker fees from Gilead. The other authors declare no conflicts of interest.

\section{Acknowledgements}

The authors acknowledge and thank Yew Sok-Wee for her assistance with the administrative cost data. The authors thank the Independent Hospital Pricing Authority for making national-level data (used to inform the modelled predictions of the studied protocol) readily available. Michelle Tew is jointly supported by the National Health and Medical Research Council of Australiafunded Centre for Research Excellence in Total Joint Replacement (1116325) and Centre for Improving Cancer Outcomes Through Enhanced Infection Services (1116876) and a Melbourne Research Scholarship. This work did not receive any direct funding.

\section{References}

1 Hughes WT, Armstrong D, Bodey GP, Bow EJ, Brown AE, Calandra T, Feld R, Pizzo PA, Rolston KV, Shenep JL, Young LS. 2002 guidelines for the use of antimicrobial agents in neutropenic patients with cancer. Clin Infect Dis 2002; 34: 730-51. doi:10.1086/339215

2 Klastersky J, Paesmans M, Rubenstein EB, Boyer M, Elting L, Feld R, Gallagher J, Herrstedt J, Rapoport B, Rolston K, Talcott J. The Multinational Association for Supportive Care in Cancer risk index: a multinational scoring system for identifying low-risk febrile neutropenic cancer patients. J Clin Oncol 2000; 18: 3038-51. doi:10.1200/ JCO.2000.18.16.3038

3 Vidal L, Ben dor I, Paul M, Eliakim-Raz N, Pokroy E, Soares-Weiser K, Leibovici L. Oral versus intravenous antibiotic treatment for febrile neutropenia in cancer patients. Cochrane Database Syst Rev 2013; 10: CD003992. doi:10.1002/14651858.CD003992.pub3

4 Vidal L, Paul M, Ben dor I, Soares-Weiser K, Leibovici L. Oral versus intravenous antibiotic treatment for febrile neutropenia in cancer patients: a systematic review and meta-analysis of randomized trials. J Antimicrob Chemother 2004; 54: 29-37. doi:10.1093/jac/dkh303

5 Carstensen M, Sorensen J. Outpatient management of febrile neutropenia: time to revise the present treatment strategy. J Support Oncol 2008; 6: 199-208.

6 Cooksley T, Campbell G, Al-Sayed T, LaMola L, Berman R. A novel approach to improving ambulatory outpatient management of low risk febrile neutropenia: an Enhanced Supportive Care (ESC) clinic. Support Care Cancer 2018; 26: 2937-2940. doi:10.1007/s00520-018-4194-1

7 Ying FLM, Ping MCY, Tong M, Yan EYP, Yee TLS, Ting LY, Sim AL, Yu LC, Shiu BL, Kin AC. A cohort study on protocol-based nurse-led out-patient management of post-chemotherapy low-risk febrile neutropenia. Support Care Cancer 2018; 26: 3039-3045. doi:10.1007/ s00520-018-4157-6

8 Elting LS, Lu C, Escalante CP, Giordano SH, Trent JC, Cooksley C, Avritscher EB, Shih YC, Ensor J, Bekele BN, Gralla RJ. Outcomes and cost of outpatient or inpatient management of 712 patients with febrile neutropenia. J Clin Oncol 2008; 26: 606-11. doi:10.1200/JCO.2007. 13.8222

9 Teuffel O, Cheng S, Ethier M, Diorio C, Martino J, Mayo C, Wing R, Sung L, Alibhai SM. Health-related quality of life anticipated with different management strategies for febrile neutropenia in adult cancer patients. Support Care Cancer 2012; 20: 2755-64. doi:10.1007/ s00520-012-1397-8

10 Lingaratnam S, Worth LJ, Slavin MA, Bennett CA, Kirsa SW, Seymour JF, Dalton A, Koczwara B, Prince HM, O'Reilly M, Mileshkin L. A cost analysis of febrile neutropenia management in Australia: ambulatory $v$. in-hospital treatment. Aust Health Rev 2011; 35: 491-500. doi:10.1071/ AH10951

11 Teh BW, Brown C, Joyce T, Worth LJ, Slavin MA, Thursky KA. Safety and cost benefit of an ambulatory program for patients with low-risk neutropenic fever at an Australian centre. Support Care Cancer 2018; 26: 997-1003. doi:10.1007/s00520-017-3921-3 
12 Teuffel O, Amir E, Alibhai S, Beyene J, Sung L. Cost effectiveness of outpatient treatment for febrile neutropaenia in adult cancer patients. $\mathrm{Br} J$ Cancer 2011; 104: 1377-83. doi:10.1038/bjc.2011.101

13 Klastersky J, Paesmans M. The Multinational Association for Supportive Care in Cancer (MASCC) risk index score: 10 years of use for identifying low-risk febrile neutropenic cancer patients. Support Care Cancer 2013; 21: 1487-95. doi:10.1007/s00520-013-1758-y

14 Innes HE, Smith D, O'Reilly S, Clark P, Kelly V, Marshall E. Oral antibiotics with early hospital discharge compared with in-patient intravenous antibiotics for low-risk febrile neutropenia in patients with cancer: a prospective randomised controlled single centre study. $\mathrm{Br} J$ Cancer 2003; 89: 43-9. doi:10.1038/sj.bjc.6600993

15 Hocking C, Taylor A, Hayward A. Early discharge and ambulatory care of low-risk patients with neutropenic fever in Australia. Intern Med J 2013; 43: 591-5. doi:10.1111/imj.12117

16 Klastersky J, Paesmans M. Risk-adapted strategy for the management of febrile neutropenia in cancer patients. Support Care Cancer 2007; 15: 477-82. doi:10.1007/s00520-006-0185-8

17 Freifeld A, Sankaranarayanan J, Ullrich F, Sun J. Clinical practice patterns of managing low-risk adult febrile neutropenia during cancer chemotherapy in the USA. Support Care Cancer 2008; 16: 181-91. doi:10.1007/s00520-007-0308-x

18 Day E, Kim S, Hughes-Davies L. Barriers to protocol-led early discharge of low-risk febrile neutropenia patients. Clin Oncol 2014; 26: 516. doi:10.1016/j.clon.2014.05.003

19 Lingaratnam S, Slavin M, Mileshkin L, Solomon B, Burbury K, Seymour J, Sharma R, Koczwara B, Kirsa SW, Davis ID, Prince M. An Australian survey of clinical practices in management of neutropenic fever in adult cancer patients 2009. Intern Med $J$ 2011; 41: 110-20. doi:10.1111/j.1445-5994.2010.02342.x

20 Independent Hospital Pricing Authority. National hospital cost data collection, public hospitals cost report, round 20 (financial year 2015-16). 2018. Available at: https://www.ihpa.gov.au/publications/national-hospital-cost-data-collection-public-hospitals-cost-report-round-200 [verified 1 August 2018].

21 Austin PC, Rothwell DM, Tu JV. A comparison of statistical modeling strategies for analyzing length of stay after CABG surgery. Health Serv Outcomes Res Methodol 2002; 3: 107-33. doi:10.1023/ A:1024260023851

22 Glick HA, Doshi JA, Sonnad SS, Polsky D. Economic evaluation in clinical trials. Oxford: Oxford University Press; 2014.

23 Lingaratnam S, Thursky K, Slavin M, Kirsa S, Bennett C, Worth L. The disease and economic burden of neutropenic fever in adult patients in
Australian cancer treatment centres 2008: analysis of the Victorian Admitted Episodes Dataset. Intern Med $J$ 2011; 41: 121-9. doi:10.1111/j.1445-5994.2010.02343.x

24 Lingaratnam S, Mellerick A, Worth L, Green M, Guy S, Kirsa S, Slavin M, Renwick W, Filshie R, Thursky KA. Feasibility of early discharge strategies for neutropenic fever: outcomes of a Victorian organisational readiness assessment and pilot. Intern Med $J$ 2013; 43: 979-86. doi:10.1111/imj.12228

25 Australian Bureau of Statistics. 6401.0 - Consumer Price Index, Australia.2018. Available at: http://www.abs.gov.au/ausstats/abs@.nsf/mf/ 6401.0 [verified 10 August 2018].

26 Australian Government Department of Health. Guidelines for preparing submissions to the Pharmaceutical Benefits Advisory Committee (PBAC). 2016. Available at: https://pbac.pbs.gov.au/ [verified 10 August 2018].

27 Australian Government Department of Health. Schedule of Pharmaceutical Benefits summary of changes effective 1 May 2017. 2017. Available at: http://www.pbs.gov.au/publication/schedule/2017/05/2017-0501-general-soc.pdf [verified 3 August 2018].

28 Australian Government Department of Health. Medicare Benefits schedule book operating from 01 May 2017. 2017. Available at: http://www. mbsonline.gov.au/internet/mbsonline/publishing.nsf/Content/ED288 42309B2CD13CA2580F7008294BE/\$File/201705-MBS.pdf [verified 3 August 2018].

29 AMA Victoria. Victorian public health sector medical specialists enterprise agreement 2013. 2013. Available at: https://northerndoctors. org.au/wp-content/uploads/2017/12/AMA-Vict-Public-Health-SectorMedical-Specialists-Enterprise-Agreemenet-2013.pdf [verified 13 August 2018].

30 AMA Victoria. Nurses and midwives (Victorian public health sector) (single interest employers) enterprise agreement 2016-2020. 2016. Available at: http://www.anmfvic.asn.au/ /media/files/ANMF/EBA\% 202016/Nurses-and-Midwives-Vic-PS-SIE-EA-2016-2020-amended [verified 13 August 2018].

31 Dulisse B, Li X, Gayle JA, Barron RL, Ernst FR, Rothman KJ, Legg JC, Kaye JA. A retrospective study of the clinical and economic burden during hospitalizations among cancer patients with febrile neutropenia. $J$ Med Econ 2013; 16: 720-35. doi:10.3111/ 13696998.2013.782034

32 Rolston KV. Neutropenic fever and sepsis: evaluation and management. In: Stosor V, Zembower T, editors. Infectious Complications in Cancer Patients. Cancer Treatment and Research, vol. 161. Cham: Springer; 2014. pp. 181-202. 